\title{
Accelerated knee osteoarthritis is associated with pre-radiographic degeneration of the extensor mechanism and cruciate ligaments: data from the Osteoarthritis Initiative
}

\author{
Julie E. Davis ${ }^{1}$, Matthew S. Harkey ${ }^{1,2}$, Robert J. Ward ${ }^{3}$, James W. MacKay ${ }^{4}$, Bing Lu ${ }^{5}$, Lori Lyn Price ${ }^{6,7}$,
} Charles B. Eaton ${ }^{8}$, Grace H. Lo ${ }^{9,10}$, Mary F. Barbe ${ }^{11}$, Timothy E. McAlindon ${ }^{1}$ and Jeffrey B. Driban ${ }^{{ }^{*}}$ (D)

\begin{abstract}
Background: To determine if adults with incident accelerated knee osteoarthritis (KOA) are more likely to have degenerative knee ligaments or tendons compared to individuals with typical or no KOA.

Methods: We identified 3 sex-matched groups among Osteoarthritis Initiative participants who had a knee without radiographic KOA at baseline (Kellgren-Lawrence $[\mathrm{KL}]<2$ ): 1 ) accelerated $\mathrm{KOA}$ : at least 1 knee had $\mathrm{KL}$ grade $\geq 3$ in $\leq 48$ months, 2) typical KOA: at least 1 knee increased in radiographic scoring within 48 months, 3) no KOA: both knees had the same $K L$ grade at baseline and 48 months. We evaluated knee magnetic resonance images up to 2 years before and after a visit when the accelerated or typical KOA criteria were met (index visit). Radiologists reported degenerative signal changes for cruciate and collateral ligaments, and extensor mechanism and proximal gastrocnemius tendons. We used generalized linear mixed models with 2 independent variables: group and time.

Results: Starting at least 2 years before onset, adults with accelerated KOA were twice as likely to have degenerative cruciate ligaments than no $\mathrm{KOA}$ (odds ratio $=2.10,95 \% \mathrm{Cl}=1.18,3.74$ ). A weaker association (not statistically significant) was detected for adults with accelerated versus typical $K O A(O R=1.72,95 \% \mathrm{Cl}=0.99,3.02)$. Regardless of time, adults with accelerated (odds ratio $=2.13$ ) or typical KOA (odds ratio $=2.16$ ) were twice as likely to have a degenerative extensor mechanism than no KOA. No other structural features were statistically significant.

Conclusions: Degenerative cruciate ligaments or extensor mechanism antedate radiographic onset of accelerated $\mathrm{KOA}$. Hence, knee instability may precede accelerated KOA, which might help identify patients at high-risk for accelerated KOA and novel prevention strategies.
\end{abstract}

Keywords: Epidemiology, Knee, Osteoarthritis, Ligaments, Tendons

\footnotetext{
* Correspondence: jeffrey.driban@tufts.edu

${ }^{1}$ Division of Rheumatology, Allergy \& Immunology, Tufts Medical Center, 800

Washington Street, Box \#406, Boston, MA 02111, USA

Full list of author information is available at the end of the article
}

(c) The Author(s). 2019 Open Access This article is distributed under the terms of the Creative Commons Attribution 4.0 International License (http://creativecommons.org/licenses/by/4.0/), which permits unrestricted use, distribution, and reproduction in any medium, provided you give appropriate credit to the original author(s) and the source, provide a link to the Creative Commons license, and indicate if changes were made. The Creative Commons Public Domain Dedication waiver (http://creativecommons.org/publicdomain/zero/1.0/) applies to the data made available in this article, unless otherwise stated. 


\section{Background}

While knee osteoarthritis (KOA) is characteristically a slow progressing disorder, there is a subset of individuals that experience an abrupt and accelerated onset of radiographic advanced-stage disease, often less than 12 months [1-3]. Individuals that develop accelerated KOA report greater knee symptoms and functional limitations than those that develop the more common and gradual onset of KOA as early as 3 years before radiographic disease onset [2]. Additionally, those with accelerated KOA are 25 times more likely to receive a knee replacement over 9 years than those with a gradual onset of KOA [4]. Furthermore, the median time from the first evidence of radiographic progression to knee replacement is only 2.3 years, which highlights the short window for tertiary prevention [4]. It is critical that we understand the pathogenesis of accelerated KOA to develop strategies to identify high-risk patients, those with early-stage (preradiographic) accelerated $\mathrm{KOA}$, and novel prevention strategies.

Degeneration of knee tendons and ligaments may antedate the onset of radiographic accelerated KOA and contribute to prodromal joint symptoms that are common to accelerated KOA. Degeneration of the cruciate ligaments are present in knees with $\mathrm{KOA}$ and may antedate KOA [5, 6]. Early degeneration of ligaments and tendons may be a sign of abnormal joint loading or instability, which increases shear force to the articular cartilage, may be an early sign of a person at risk for $\mathrm{OA}$, and a risk factor for a knee injury that could precipitate accelerated KOA [7]. However, it remains unclear if the degenerative status of the ligaments and tendons in the knee are associated with the development of accelerated KOA.

We aimed to determine if incident accelerated KOA is preceded and characterized by the degeneration of cruciate or collateral ligaments, as well as extensor mechanism or proximal gastrocnemius tendons compared to those with no KOA or a gradual onset of KOA (typical KOA). The Osteoarthritis Initiative (OAI) offers a unique opportunity to study the relationship between degeneration of ligaments and tendons and the natural history of incident $\mathrm{KOA}$, including accelerated $\mathrm{KOA}$. If people with accelerated KOA exhibit unique degenerative ligament or tendon characteristics leading to the onset of disease, it may indicate accelerated KOA is preceded by maladaptive responses to overloading or instability. If so, this may help identify high-risk patients and novel prevention strategies.

\section{MethodS}

We conducted a longitudinal analysis of individuals in the Osteoarthritis Initiative (OAI) using radiographs, magnetic resonance (MR) images, and clinical data at baseline and the first 4 annual follow-up visits. The OAI is a multicenter cohort study of 4796 adults with or at risk for symptomatic KOA. Staff at four clinical sites (Memorial Hospital of Rhode Island, University of Maryland and Johns Hopkins University, the University of Pittsburgh, and The Ohio State University) recruited participants between 2004 and 2006. OAI data (including images) and protocols are publicly available [8]. The OAI has met all criteria for ethical standards regarding human studies as described in the 1964 Declaration of Helsinki and all amendments. The OAI study was reviewed and approved by institutional review boards at each OAI clinical site and the OAI coordinating center (Memorial Hospital of Rhode Island Institutional Review Board, University of Maryland Baltimore - Institutional Review Board, University of Pittsburgh Institutional Review Board, and The Ohio State University's Biomedical Sciences Institutional Review Board; Committee on $\mathrm{Hu}$ man Research at University of California, San Francisco; Approval Number: 10-00532). All participants offered written informed consent before starting the study.

\section{Participant selection}

We classified 3 groups based on radiographic criteria. Adults with incident accelerated KOA included anyone with at least 1 knee that had no radiographic $\mathrm{KOA}$ at baseline (Kellgren-Lawrence $[\mathrm{KL}]$ grade $<2$ ) that developed advanced-stage KOA (KL grade 3 or 4) within 48 months $(n=125)[3,9]$. Individuals with typical KOA had no baseline radiographic KOA $(\mathrm{KL}<2)$ in both knees and 1 or more knee increased in radiographic scoring within 48 months $(n=187$; excluding those with accelerated KOA). Individuals with no KOA had no radiographic KOA in both knees at baseline and had no change in KL grade in either knee during 48-months $(n=1325)$.

Our overall goal of the accelerated KOA project was to characterize accelerated $\mathrm{KOA}$ using imaging and biochemical biomarkers as well as an array of clinical outcomes (e.g., patient-reported outcomes, functional assessments). Hence, to ensure the feasibility of completing all of the biomarker assessments we did 1:1:1 matching based on sex ( $n=125$ participants/group).

\section{Index knee}

For adults with incident accelerated or typical KOA, the index knee was the first knee to experience accelerated or typical KOA, respectively. For an individual with no KOA, we defined the index knee to be the same side as their matched member of the accelerated KOA group.

\section{Definition of index visit}

For individuals with accelerated KOA, the index visit was the OAI visit when a participant met the criteria for 
accelerated KOA (i.e., visit when someone had $\mathrm{KL}=3$ or 4; potential OAI visits: 12-, 24-, 36-, or 48-month visit). For adults with typical KOA, the index visit was the OAI visit when a participant met the criteria for typical KOA (i.e., visit when someone first increased KL grade; potential OAI visits: 12-, 24-, 36-, or 48-month visit). For someone with no KOA, the index visit was the same OAI visit as that person's matched member of the incident accelerated KOA group. Figure 1 illustrates examples of the index visit and observation period for a member of each group.

\section{Knee radiographs}

To classify individuals into groups we relied on readings of bilateral weight-bearing, fixed-flexion posteroanterior knee radiographs. These images were obtained at baseline and annual follow-up visits. Blinded central readers recorded KL grades ( 0 to 4 ; weighted kappa $=0.70-0.80$; files: kXR_SQ_BU\#\#_SAS; file versions: 0.6, 1.6, 3.5, 5.5, and 6.3) [8].

\section{Magnetic resonance imaging}

Magnetic resonance (MR) images were attained annually with one of four identical Siemens (Erlangen, Germany) Trio 3-Tesla MR systems at each OAI site. The musculoskeletal radiologists (RJW and JWM) performing semiquantitative scoring were provided all the sequences acquired on each index knee at each visit; including a sagittal intermediate-weighted, turbo spin echo, fatsuppressed MR sequence with the following parameters: field of view $=160 \mathrm{~mm}$, slice thickness $=3 \mathrm{~mm}$, skip $=0$ $\mathrm{mm}$, flip angle $=180$ degrees, echo time $=30 \mathrm{~ms}$, recovery time $=3200 \mathrm{~ms}, 313 \times 448$ matrix, $\mathrm{x}$ resolution $=$ $0.357 \mathrm{~mm}$, y resolution $=0.511 \mathrm{~mm}$, and total slice number $=37$. The OAI sequences have been described elsewhere $[8,10]$.

\section{MR image readings}

Two musculoskeletal radiologists reviewed MR images (RJW:255 cases, JWM:120 cases) to assess the integrity of the anterior and posterior cruciate ligaments, medial and lateral collateral ligaments, extensor mechanism (quadriceps femoris tendon and patellar ligament) [11], and gastrocnemius proximal tendons. Readers noted if the structures appeared normal or had degenerative pathologic findings. We defined a degenerative appearance as the existence of atypical intrinsic high-signal intensity in the substance of the ligaments or tendon without a discrete tear. Readers had good agreement among 25 cases: prevalence-adjusted and bias-adjusted kappa (PABAK) were 0.42 to 0.75 : anterior cruciate ligament $\mathrm{PABAK}=0.50$, posterior cruciate ligament $\mathrm{PABAK}=0.48$, medial collateral ligament $\mathrm{PABAK}=0.75$, lateral collateral ligament $\mathrm{PABAK}=0.67$, extensor mechanism $\mathrm{PABAK}=0.42$, gastrocnemius proximal tendons PABAK $=0.42$.

Since less than $10 \%$ of adults with no KOA $(n<6)$ had degeneration in the posterior cruciate ligament we analyzed the cruciate ligaments collectively as one outcome: cruciate ligament degeneration, which was defined as

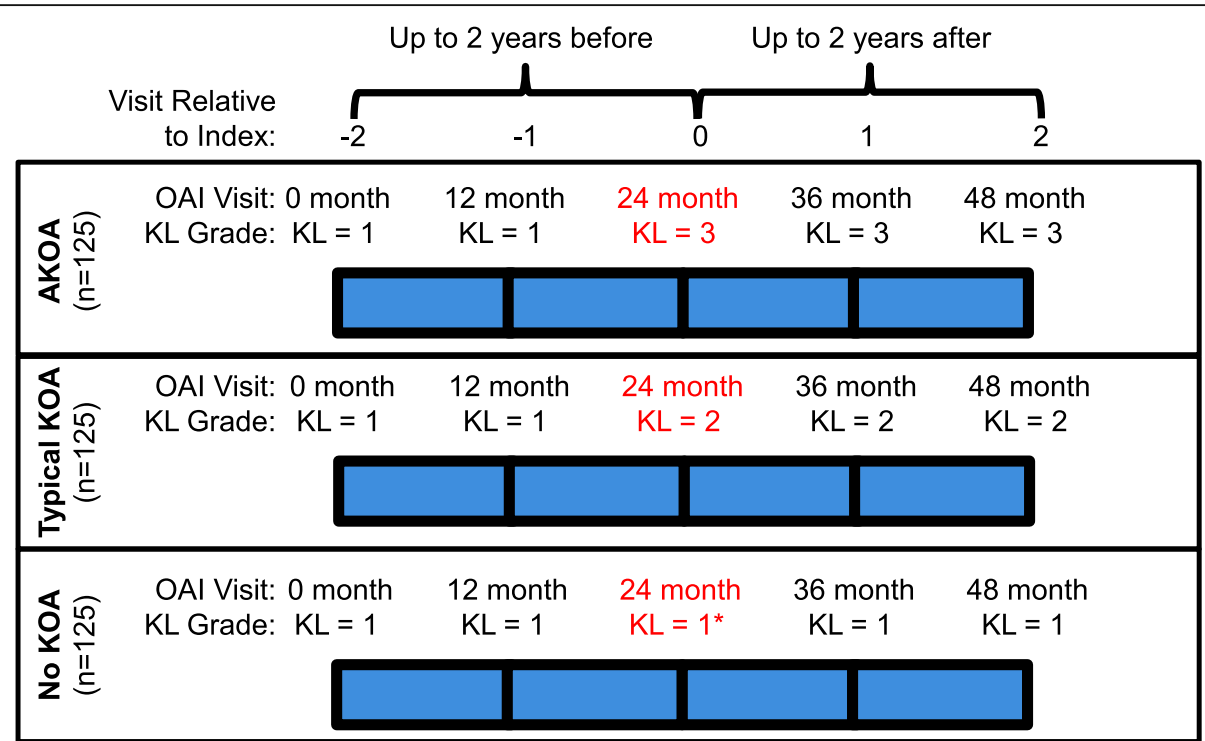

Fig. 1 Examples of the Index Visit and Observation Period for a Member of Each Group. Key abbreviations: Accelerated Knee Osteoarthritis (AKOA), Knee Osteoarthritis (KOA), or No KOA. Kellgren-Lawrence (KL), Osteoarthritis Initiative (OAI). The red font represents the index visit (visit relative to index $=0$ ). For individuals with accelerated or typical KOA, the index visit was the OAI visit when a person met the criteria for accelerated or typical KOA, respectively. * For someone with no KOA, the index visit was the same visit as that person's matched member of the incident accelerated KOA group. The observation period was up to 2 years before and after the index visit (visit relative to index $=-2$ to 2 years) 
the presence of degenerative appearance in the anterior or posterior cruciate ligament. Similarly, less than $10 \%$ of adults with no KOA $(n<3)$ had degeneration in the lateral collateral ligament; hence, we analyzed the collateral ligaments collectively as one outcome: collateral ligament degeneration, which was defined as the presence of degenerative appearance in the medial or lateral collateral ligament.

\section{Statistical analysis}

We ran descriptive statistics to assess baseline characteristics of the 3 groups. To determine any association between degeneration of the ligaments or tendons and group assignment, we used generalized linear mixed models assuming compound symmetry. Independent variables included group (3 levels), and time (up to 5 levels). We also assessed a group-by-time interaction. For our primary analyses we adjusted for sex (matching variable) [12] and factors associated with missing $M R$ data at the next visit (i.e., age, body mass index, injury, frequent knee pain, days with limited activity in prior month, overall global rating, and WOMAC pain).

We conducted several sensitivity analyses: 1) adjusted only for sex, which was our matching factor, 2) among people who developed accelerated KOA and had no radiographic OA bilaterally at baseline $(n=54$ per group with matched sample), 3) among individuals who developed accelerated KOA in $<12$ months ( $n=71$ per group with matched sample), and 4) among those who developed typical $\mathrm{KOA}$ and had $\mathrm{KL}=2$, which is a common radiographic criteria for KOA $(n=76$ per group with matched sample). We also performed one sensitivity analysis with no matching among people with complete data at every visit (accelerated KOA $n=12$, typical KOA $n=29$, no KOA $n=25$ ). Since the sensitivity analyses were always among a smaller sample size, we performed them adjusted for sex only. All analyses were conducted with SAS Enterprise 7.15 (Cary, NC, USA).

\section{Results}

\section{Descriptive characteristics}

Table 1 offers an overview of the characteristics of the three groups. In brief, the groups were mostly female (63\%), overweight, and 24 to $39 \%$ of participants in each group reported frequent knee pain during the prior 12 months.

Table 2 highlights the frequency of the degenerative ligaments and tendons over time.

We detected a significant group effect - but no groupby-time interaction - for the presence of degenerative cruciate ligaments (group $p=0.03$; interaction $p=0.24$ ) and degenerative extensor mechanism (group $p=0.01$; interaction $p=0.43$ ). We observed no significant associations with group nor group-by-time interaction with the presence of degenerative collateral ligaments (group $p=0.43$; interaction $p=0.06$ ) and proximal gastrocnemius tendon degeneration (group $p=0.71$; interaction $p=0.12$ ).

Starting at 2 years prior to the index visit, the presence of degenerative cruciate ligaments was stable over time: ranging from 43 to $51 \%$ among those with accelerated KOA, 22 to $33 \%$ among those with typical KOA, and 21 to $26 \%$ among people with no KOA. Regardless of time, adults with accelerated KOA were twice as likely to have degenerative cruciate ligaments than those with no KOA (odds ratio $[\mathrm{OR}]=2.10,95 \%$ confidence interval $[\mathrm{CI}]=$ 1.18 to 3.74). We found a weaker association, which was not statistically significant, for those with accelerated $\mathrm{KOA}$ versus those with typical $\mathrm{KOA}(\mathrm{OR}=1.73,95 \%$ $\mathrm{CI}=0.99$ to 3.02 ). Adults with typical KOA had a similar odds of having a degenerative cruciate ligament as those with no $\mathrm{KOA}(\mathrm{OR}=1.21,95 \% \mathrm{CI}=0.68$ to 2.17$)$. The sensitivity analyses supported an association between degenerative cruciate ligaments and accelerated KOA (accelerated KOA vs No KOA OR range: 2.59 to 6.28; accelerated KOA vs typical KOA OR range: 1.68 to 5.38 ) with the highest OR among participants with complete data. Similarly, the sensitivity analyses typically

Table 1 Descriptive Characteristics of those with Accelerated, Common, and No Knee Osteoarthritis (KOA) at Baseline

\begin{tabular}{|c|c|c|c|}
\hline $\begin{array}{l}\text { Variables } \\
\text { (means, SD; except where noted) }\end{array}$ & $\begin{array}{l}\text { AKOA } \\
(n=125)\end{array}$ & $\begin{array}{l}\text { Typical KOA } \\
(n=125)\end{array}$ & $\begin{array}{l}\text { No KOA } \\
(n=125)\end{array}$ \\
\hline Females (n, \%) & $79(63 \%)$ & $79(63 \%)$ & $79(63 \%)$ \\
\hline Index knee KL Grade $=0(n, \%)$ & $42(34 \%)$ & $71(57 \%)$ & $92(74 \%)$ \\
\hline Patellofemoral Osteoarthritis (MR-based) & $88(75 \%)$ & $84(69 \%)$ & $80(66 \%)$ \\
\hline Frequent knee pain in past 12 months $(\mathrm{n}, \%)$ & $44(35 \%)$ & $49(39 \%)$ & $30(24 \%)$ \\
\hline Age (years) & $62.5(8.5)$ & $58.4(8.4)$ & $57.3(8.2)$ \\
\hline Body mass index $\left(\mathrm{kg} / \mathrm{m}^{2}\right)$ & $29.7(4.6)$ & $28.1(4.4)$ & $26.9(4.4)$ \\
\hline Global impact rating ( 0 to 10 ; higher score $=$ greater impact $)$ & $1.7(1.9)$ & $1.1(1.5)$ & $0.8(1.1)$ \\
\hline How many days limited activities in past 30 days ( 0 to 30)? & $3.2(7.3)$ & $1.7(4.8)$ & $1.4(4.3)$ \\
\hline WOMAC pain (0 to 20 ; higher score $=$ more pain) & $2.3(3.1)$ & $1.8(2.3)$ & $1.6(2.4)$ \\
\hline
\end{tabular}

AKOA accelerated knee osteoarthritis, KL Kellgren-Lawrence, MR magnetic resonance, WOMAC Western Ontario and McMaster Universities Osteoarthritis Index 
Table 2 Frequency of Degenerative Ligaments/Tendons Among Accelerated Knee Osteoarthritis (AKOA), Common Knee Osteoarthritis (KOA), No KOA

\begin{tabular}{|c|c|c|c|c|}
\hline Frequency (n [\%]) & Visit & AKOA & Typical KOA & No KOA \\
\hline Cruciate Ligaments & -2 & $39(43)$ & $14(22)$ & $25(26)$ \\
\hline \multirow[t]{4}{*}{ (Anterior \& Posterior) } & -1 & $50(43)$ & $34(28)$ & $30(24)$ \\
\hline & Index & $49(47)$ & $35(28)$ & $30(24)$ \\
\hline & 1 & $41(51)$ & $33(31)$ & $25(26)$ \\
\hline & 2 & $17(47)$ & $27(33)$ & $11(21)$ \\
\hline \multirow{5}{*}{$\begin{array}{l}\text { Anterior Cruciate } \\
\text { Ligament }^{\mathrm{a}}\end{array}$} & -2 & $36(40)$ & $14(22)$ & $23(24)$ \\
\hline & -1 & $44(38)$ & $32(26)$ & $27(22)$ \\
\hline & Index & $44(42)$ & $33(27)$ & $27(22)$ \\
\hline & 1 & $35(44)$ & $31(28)$ & $22(23)$ \\
\hline & 2 & $15(42)$ & $24(29)$ & $9(17)$ \\
\hline \multirow{5}{*}{$\begin{array}{l}\text { Posterior Cruciate } \\
\text { Ligament }^{a}\end{array}$} & -2 & $9(10)$ & $1(2)$ & $5(5)$ \\
\hline & -1 & $14(12)$ & $8(6)$ & $6(5)$ \\
\hline & Index & $13(12)$ & $8(6)$ & $6(5)$ \\
\hline & 1 & $12(15)$ & $7(6)$ & $6(6)$ \\
\hline & 2 & $6(17)$ & $8(10)$ & $3(6)$ \\
\hline Collateral Ligaments & -2 & $22(24)$ & $6(9)$ & $12(13)$ \\
\hline \multirow[t]{4}{*}{ (Medial \& Lateral) } & -1 & $28(24)$ & $19(15)$ & $17(14)$ \\
\hline & Index & $28(26)$ & $19(15)$ & $17(14)$ \\
\hline & 1 & $24(30)$ & $20(18)$ & $15(15)$ \\
\hline & 2 & $8(22)$ & $17(20)$ & $8(15)$ \\
\hline \multirow{5}{*}{$\begin{array}{l}\text { Medial Collateral } \\
\text { Ligament }^{\mathrm{a}}\end{array}$} & -2 & $19(21)$ & $5(8)$ & $11(11)$ \\
\hline & -1 & $24(11)$ & $16(13)$ & $16(13)$ \\
\hline & Index & $24(23)$ & $16(13)$ & $16(13)$ \\
\hline & 1 & $23(29)$ & $17(15)$ & $14(14)$ \\
\hline & 2 & $8(22)$ & $14(17)$ & $8(15)$ \\
\hline \multirow{5}{*}{$\begin{array}{l}\text { Lateral Collateral } \\
\text { Ligament }^{a}\end{array}$} & -2 & $5(5)$ & $3(5)$ & $3(3)$ \\
\hline & -1 & $7(6)$ & $6(5)$ & $3(2)$ \\
\hline & Index & $7(7)$ & $6(5)$ & $3(2)$ \\
\hline & 1 & $4(5)$ & $6(5)$ & $2(2)$ \\
\hline & 2 & $2(6)$ & $6(7)$ & $0(0)$ \\
\hline \multirow[t]{5}{*}{ Extensor Mechanism } & -2 & $40(43)$ & $26(40)$ & $21(22)$ \\
\hline & -1 & $50(43)$ & $48(39)$ & $30(24)$ \\
\hline & Index & $47(45)$ & $51(41)$ & $32(26)$ \\
\hline & 1 & $36(46)$ & $46(42)$ & $26(27)$ \\
\hline & 2 & $11(31)$ & $33(39)$ & $17(32)$ \\
\hline \multirow{5}{*}{$\begin{array}{l}\text { Proximal Gastrocnemius } \\
\text { Tendon }\end{array}$} & -2 & $46(50)$ & $25(38)$ & $39(41)$ \\
\hline & -1 & $54(46)$ & $54(44)$ & $51(41)$ \\
\hline & Index & $50(47)$ & $56(45)$ & $51(41)$ \\
\hline & 1 & $37(46)$ & $50(45)$ & $41(42)$ \\
\hline & 2 & $15(42)$ & $40(48)$ & 20 (38) \\
\hline
\end{tabular}

${ }^{a}$ The sum of the prevalence for each specific ligaments will not equal the overall cruciate or collateral ligament prevalence because some knees had anterior and posterior cruciate ligament degeneration or medial and lateral collateral ligament degeneration supported the finding that degenerative cruciate ligaments had a weak or no association with typical KOA (typical KOA vs No KOA OR range: 0.66 to 1.22 ) with the exception of a potential association among those who developed typical KOA with $\mathrm{KL}=2(\mathrm{OR}=1.85,95 \%$ $\mathrm{CI}=0.88$ to 3.92 ).

Starting at 2 years prior to the index visit, the presence of degenerative extensor mechanism was stable over time: ranging from 31 to $43 \%$ among those with accelerated KOA, 39 to $42 \%$ among those with typical KOA, and 22 to $32 \%$ among people with no KOA. Regardless of time, adults with accelerated or typical KOA were twice as likely to have degenerative extensor mechanism than those with no KOA (accelerated KOA vs no KOA $\mathrm{OR}=2.13,95 \% \mathrm{CI}=1.19$ to 3.82 ; typical $\mathrm{KOA}$ vs no $\mathrm{KOA} \mathrm{OR}=2.16,95 \% \mathrm{CI}=1.23$ to 3.79 ). Neither people with accelerated nor typical KOA were more likely to have a degenerative extensor mechanism than their peers (accelerated vs typical $\mathrm{KOA} \mathrm{OR}=0.99,95 \% \mathrm{CI}=$ 0.57 to 1.70 ). The sensitivity analyses showed similar results (OR accelerated KOA vs No KOA OR range: 1.37 to 2.88; typical KOA vs No KOA OR range: 1.32 to 2.92; accelerated KOA vs typical KOA OR range: 0.99 to 1.50 )

\section{Discussion}

Adults with incident KOA (accelerated or typical KOA) were more likely to have a degenerative extensor mechanism than those with no KOA. Furthermore, adults with accelerated KOA were more likely to present with degenerative cruciate ligaments than those with no $\mathrm{KOA}$, regardless of time. The degenerative changes were evident at least 2 years prior to meeting the criteria for incident disease and remained stable over time. Hence, these changes may be early signs that can extend our understanding of the pathogenesis of $\mathrm{KOA}$, especially accelerated KOA. These findings may offer novel insights to recognize adults at-risk for or with early-stage accelerated KOA and help identify prevention strategies.

The current findings supported previous observations that there is an association between degenerative cruciate ligaments and symptomatic KOA within the OAI [13]. However, our results expanded on those findings by demonstrating that cruciate ligament degeneration was more associated with incident accelerated KOA than a gradual onset of KOA. The cruciate ligament degeneration, which is characterized by altered fiber arrangement and collagen composition, may compromise ligamentous function and lead to instability and altered joint loading [5, 14]. In fact, a degenerative cruciate ligament may have similar consequences to a joint as a complete tear. For example, individuals with ACL degeneration and individuals with an ACL rupture presented with a greater severity of structural pathology (e.g., 
cartilage damage, bone marrow lesions) when compared to individuals with a normal ACL; however, there were no differences in these pathologies between adults with degenerative or acute ACL pathology [7]. These structural changes may be attributable to rotational and antero-posterior instability and increased external adduction moments that occur during walking $[15,16]$. These biomechanical changes may increase shear forces and medial compartment loading, which could contribute to accelerated KOA $[15,16]$. The degenerative cruciate ligaments among those with accelerated KOA may indicate that this rotational or anteroposterior instability stresses the knee prior to the onset of disease. Additionally, cruciate ligament degeneration may also place an individual at greater risk for a knee injury, which may compound the risk of developing accelerated KOA [13]. Adults with accelerated KOA may be distinct from those with typical KOA and warrant greater consideration in both clinical and research settings [2, 17-20]. Early detection of cruciate ligament degeneration may help clinicians and researchers identify people at risk for or with early-stage accelerated KOA and enable them to implement biomechanical interventions (e.g., gait retraining, bracing) or exercise programs that reduce the risk of injury or falls (e.g. neuromuscular training).

The role of a degenerative extensor mechanism in KOA has been less studied and the significance of it among those with accelerated or typical KOA should be further explored. While multiple structures can help distribute the load during knee flexion (e.g., proximal gastrocnemius tendons), the extensor mechanism is the sole mechanism for knee extension. Therefore, more direct loading to the extensor may contribute to the development of KOA; however, the cause and clinical implications of extensor mechanism degeneration (e.g., implications for strength testing and therapeutic exercises) warrants further study.

While this study characterized which ligamentous and tendinous structures antedated the onset of accelerated KOA, we acknowledge important limitations. For example, our inter-reader agreement for detecting the presence of degenerative signal changes in the ligaments and tendons ranged from moderate to good. If we had a stronger level of inter-reader agreement, we may have observed stronger associations between these pathologic findings and accelerated or typical KOA. Despite our inter-reader agreement, we observed significant associations between degenerative extensor mechanisms and cruciate ligaments with accelerated KOA. We also had missing MR data that could effect the results, particularly 2 years after the index visit. However, we performed robust analyses that adjusted for variables related to missing MR data. We also performed several sensitivity analyses to explore the robustness of these findings. Our study was also limited by the number of adults in each group. Despite this, the OAI offered an extraordinary chance to study the natural history of accelerated KOA.

\section{Conclusions}

Starting as early as 2 years prior to radiographic onset of disease, adults with incident KOA were twice as likely to have a degenerative extensor mechanism. Furthermore, adults with accelerated KOA were more likely to have degenerative cruciate ligaments, which could be the result of excessive overloading or contribute altered joint mechanics that may increase the chance of accelerated KOA. Identifying people with these degenerative structures using MR imaging prior to the onset of KOA may help us target prevention strategies to help ameliorate the risk of KOA, especially accelerated KOA.

\section{Abbreviations}

ACL: Anterior cruciate ligament; AKOA: Accelerated knee osteoarthritis; Cl: Confidence interval; KL: Kellgren-Lawrence; KOA: Knee osteoarthritis; MR: Magnetic resonance; OAl: Osteoarthritis Initiative; OR: Odds ratio; PABAK: Prevalence-adjusted and bias-adjusted kappa; PCL: Posterior cruciate ligament

\section{Acknowledgments}

Not applicable.

\section{Authors' contributions}

JED contributed to the analysis and interpretation of data and drafting/ revising of article. RJW contributed to the conception and design, analysis and interpretation of data, and revisions of article. JWM contributed to the conception and design, analysis and interpretation of data, and revisions of article. MSH contributed to the analysis and interpretation of data and drafting/revisions of article. LLP contributed to the analysis and interpretation of data, and revisions of article. BL contributed to the conception and design, analysis and interpretation of data, and revisions of article. GHL contributed to the analysis and interpretation of data and revisions of article. CBE contributed to the conception and design, acquisition of data, analysis and interpretation of data, and revisions of article. MFB contributed to the conception and design and revisions of article. TEM contributed to the conception and design, acquisition of data, analysis and interpretation of data, and revisions of article. JBD contributed to the conception and design, analysis and interpretation of data, and drafting/revisions of article. All authors provided final approval of the version to be published and agreed to be accountable for all aspects of the work in ensuring that questions related to the accuracy or integrity of any part of the work are appropriately investigated and resolved.

\section{Funding}

These analyses were financially supported by the National Institute of Arthritis and Musculoskeletal and Skin Diseases of the National Institutes of Health under Award Number R01 AR065977. The OAl is a public-private partnership comprised of five contracts (N01-AR-2-2258; N01-AR-2-2259; N01AR-2-2260; N01-AR-2-2261; N01-AR-2-2262) funded by the National Institutes of Health, a branch of the Department of Health and Human Services, and conducted by the OAI Study Investigators. Private funding partners include Merck Research Laboratories; Novartis Pharmaceuticals Corporation, GlaxoSmithKline; and Pfizer, Inc. Private sector funding for the OAl is managed by the Foundation for the National Institutes of Health. This manuscript was prepared using an OAI public use data set and does not necessarily reflect the opinions or views of the OAl investigators, the $\mathrm{NIH}$, or the private funding partners. Dr. Lo is supported by K23 AR062127, an NIH/NIAMS funded mentored award, providing support for design and conduct of the study, analysis, and interpretation of the data. This work is supported in part with resources at the VA HSR\&D Center for Innovations in Quality, Effectiveness and Safety (\#CIN 13413), at the Michael E. DeBakey VA Medical Center, Houston, TX. The views 
expressed in this article are those of the authors and do not necessarily represent the views of the Department of Veterans Affairs. Funding bodies had no role in the design of the study and collection, analysis, and interpretation of data and in writing the manuscript.

\section{Availability of data and materials}

The datasets analyzed during current study are available in the OAl repository, https://nda.nih.gov/oai/. The MR imaging readings will be made publicly available via the OAI repository or by contacting the corresponding author.

\section{Ethics approval and consent to participate}

Institutional review boards at all OAl clinical sites and coordinating center (Memorial Hospital of Rhode Island Institutional Review Board, The Ohio State University's Biomedical Sciences Institutional Review Board, University of Pittsburgh Institutional Review Board, and University of Maryland Baltimore - Institutional Review Board, and Committee on Human Research at University of California, San Francisco; Approval Number: 10-00532) approved the study. The OAl has been approved and meets all criteria for ethical standards regarding human and animal studies defined in the 1964 Declaration of Helsinki and all amendments made after. All participants provided written informed consent prior to participation.

\section{Consent for publication}

Not applicable.

\section{Competing interests}

$\mathrm{JBD}, \mathrm{MFB}$, and MSH are members of the Editorial Board of BMC Musculoskeletal Disorders. The other authors declare that they have no competing interests.

\section{Author details}

'Division of Rheumatology, Allergy \& Immunology, Tufts Medical Center, 800 Washington Street, Box \#406, Boston, MA 02111, USA. ${ }^{2}$ Department of Population and Quantitative Health Sciences, University of Massachusetts Medical School, 368 Plantation Street, Worcester, MA 01605, USA. ${ }^{3}$ Department of Radiology, Tufts Medical Center, 800 Washington Street, Boston, MA 02111, USA. ${ }^{4}$ Department of Radiology, University of Cambridge School of Clinical Medicine, Box 218, Level 5, Addenbrooke's Hospital, Cambridge CB2 OQQ, UK. ${ }^{5}$ Brigham \& Women's Hospital and Harvard Medical School, 75 Francis Street PBB-B3, Boston, MA 02115, USA. ${ }^{6}$ The Institute for Clinical Research and Health Policy Studies, Tufts Medical Center, 800 Washington Street, Box \#63, Boston, MA 02111, USA. ${ }^{7}$ Tufts Clinical and Translational Science Institute, Tufts University, 800 Washington Street, Box \#63, Boston, MA 02111, USA. ${ }^{8}$ Center for Primary Care and Prevention, Alpert Medical School of Brown University, 111 Brewster Street, Pawtucket, RI 02860, USA. ${ }^{9}$ Medical Care Line and Research Care Line, Houston Health Services Research and Development (HSR\&D) Center of Excellence Michael E. DeBakey VAMC, Houston, TX, USA. ${ }^{10}$ Section of Immunology Allergy and Rheumatology, Baylor College of Medicine, Houston, TX. 1 Baylor Plaza, BCM-285, Houston, TX 77030, USA. "'Department of Anatomy and Cell Biology, Temple University School of Medicine, 3500 North Broad Street, Philadelphia, PA 19140, USA.

\section{Received: 25 July 2018 Accepted: 18 June 2019}

Published online: 29 June 2019

\section{References}

1. Driban JB, Eaton CB, Lo GH, Ward RJ, Lu B, McAlindon TE. Association of knee injuries with accelerated knee osteoarthritis progression: data from the osteoarthritis initiative. Arthritis Care Res (Hoboken). 2014;66(11):1673-9.

2. Driban JB, Price LL, Eaton CB, Lu B, Lo GH, Lapane KL, McAlindon TE. Individuals with incident accelerated knee osteoarthritis have greater pain than those with common knee osteoarthritis progression: data from the osteoarthritis initiative. Clin Rheumatol. 2016;35(6):1565-71.

3. Driban JB, Stout AC, Lo GH, Eaton CB, Price LL, Lu B, Barbe MF, McAlindon TE. Best performing definition of accelerated knee osteoarthritis: data from the osteoarthritis initiative. Ther Adv Musculoskelet Dis. 2016;8(5):165-71.

4. Davis JE, Liu SH, Lapane K, Harkey MS, Price LL, Lu B, Lo GH, Eaton CB, Barbe MF, McAlindon TE, et al. Adults with incident accelerated knee osteoarthritis are more likely to receive a knee replacement: data from the osteoarthritis initiative. Clin Rheumatol. 2018;37(4):1115-8.

5. Hasegawa A, Otsuki S, Pauli C, Miyaki S, Patil S, Steklov N, Kinoshita M, Koziol J, D'Lima DD, Lotz MK. Anterior cruciate ligament changes in the human knee joint in aging and osteoarthritis. Arthritis Rheum. 2012;64(3):696-704.

6. Kumagai K, Sakai K, Kusayama Y, Akamatsu Y, Sakamaki K, Morita S, Sasaki T, Saito T, Sakai T. The extent of degeneration of cruciate ligament is associated with chondrogenic differentiation in patients with osteoarthritis of the knee. Osteoarthr Cartil. 2012;20(11):1258-67.

7. Hovis KK, Alizai H, Tham S-C, Souza RB, Nevitt MC, McCulloch CE, Link TM. Non-traumatic anterior cruciate ligament abnormalities and their relationship to osteoarthritis using morphological grading and cartilage T2 relaxation times: data from the osteoarthritis initiative (OAI). Skelet Radiol. 2012:41(11):1435-43.

8. The Osteoarthritis Initiative [https://nda.nih.gov/oai/]. Accessed 24 June 2019.

9. Driban JB, Davis JE, Lu B, Price LL, Ward RJ, MacKay JW, Eaton CB, Lo GH, Barbe MF, Zhang M, Pang J, Stout AC, Harkey MS, MCAlindon TE. Accelerated Knee Osteoarthritis Is Characterized by Destabilizing Meniscal Tears and Preradiographic Structural Disease Burden. Arthritis Rheumatol. 2018. https://doi.org/10.1002/art.40826. [Epub ahead of print]. PMID: 30592385.

10. Peterfy CG, Schneider E, Nevitt M. The osteoarthritis initiative: report on the design rationale for the magnetic resonance imaging protocol for the knee. Osteoarthr Cartil. 2008;16(12):1433-41.

11. Yablon CM, Pai D, Dong Q, Jacobson JA. Magnetic resonance imaging of the extensor mechanism. Magn Reson Imaging Clin N Am. 2014; 22(4):601-20

12. Pearce N. Analysis of matched case-control studies. BMJ. 2016;352:1969.

13. Mclntyre J, Moelleken S, Tirman P. Mucoid degeneration of the anterior cruciate ligament mistaken for ligamentous tears. Skelet Radiol. 2001; 30(6):312-5.

14. Kumagai K, Sakai K, Kusayama Y, Akamatsu Y, Sakamaki K, Morita S, Sasaki T, Saito T, Sakai T. The extent of degeneration of cruciate ligament is associated with chondrogenic differentiation in patients with osteoarthritis of the knee. Osteoarthr Cartil. 2012;20(11):1258-67.

15. Almekinders LC, Pandarinath R, Rahusen FT: Knee stability following anterior cruciate ligament rupture and surgery. The contribution of irreducible tibial subluxation. J Bone Joint Surg Am. 2004;86-a(5):983-987.

16. Georgoulis AD, Papadonikolakis A, Papageorgiou CD, Mitsou A, Stergiou N. Three-dimensional tibiofemoral kinematics of the anterior cruciate ligamentdeficient and reconstructed knee during walking. Am J Sports Med. 2003; 31(1):75-9.

17. Davis J, Eaton CB, Lo GH, Lu B, Price LL, McAlindon TE, Barbe MF, Driban JB. Knee symptoms among adults at risk for accelerated knee osteoarthritis: data from the osteoarthritis initiative. Clin Rheumatol. 2017:36(5):1083-9.

18. Driban JB, McAlindon TE, Amin M, Price LL, Eaton CB, Davis JE, Lu B, Lo GH, Duryea J, Barbe MF. Risk factors can classify individuals who develop accelerated knee osteoarthritis: data from the osteoarthritis initiative. J Orthop Res. 2017

19. Driban JB, Stout AC, Duryea J, Lo GH, Harvey WF, Price LL, Ward RJ, Eaton CB, Barbe MF, Lu B, et al. Coronal tibial slope is associated with accelerated knee osteoarthritis: data from the osteoarthritis initiative. BMC Musculoskelet Disord. 2016;17:299.

20. Driban JB, Ward RJ, Eaton CB, Lo GH, Price LL, Lu B, McAlindon TE. Meniscal extrusion or subchondral damage characterize incident accelerated osteoarthritis: data from the osteoarthritis initiative. Clin Anat. 2015:28(6):792-9.

\section{Publisher's Note}

Springer Nature remains neutral with regard to jurisdictional claims in published maps and institutional affiliations. 